\title{
Spatial distribution of endemic fluorosis caused by drinking water in a high-fluorine area in Ningxia, China
}

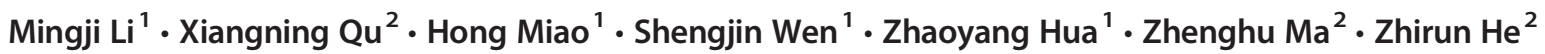

Received: 29 November 2019 / Accepted: 16 March 2020/Published online: 2 April 2020

(C) Springer-Verlag GmbH Germany, part of Springer Nature 2020

\begin{abstract}
Endemic fluorosis is widespread in China, especially in the arid and semi-arid areas of northwest China, where endemic fluorosis caused by consumption of drinking water high in fluorine content is very common. We analyzed data on endemic fluorosis collected in Ningxia, a typical high-fluorine area in the north of China. Fluorosis cases were identified in 539 villages in 1981, in 4449 villages in 2010, and in 3269 villages in 2017. These were located in 19 administrative counties. In 2017, a total of 1.07 million individuals suffered from fluorosis in Ningxia, with more children suffering from dental fluorosis and skeletal fluorosis. Among Qingshuihe River basin disease areas, the high incidence of endemic fluorosis is in Yuanzhou District and Xiji County of Guyuan City. The paper holds that the genesis of the high incidence of endemic fluorosis in Qingshui River basin is mainly caused by chemical weathering, evaporation and concentration, and dissolution of fluorine-containing rocks around the basin, which is also closely related to the semi-arid geographical region background, basin structure, groundwater chemical characteristics, and climatic conditions of the basin. The process of mutual recharge and transformation between Qingshui River and shallow groundwater in the basin is intense. There is a close coupling relationship between the power of central and southern Ningxia disease areas and Qingshui River watershed of excess fluorine water. Because the traditional drinking water source of the residents in the basin is shallow groundwater, the underground water is the main cause of the high incidence of endemic fluorosis. The results show that Xiji County and Yuanzhou District were the areas of high incidence of endemic fluorosis which gradually decreased to the middle and lower reaches of Qingshui River. In space, distribution characteristics are Qingshui River source area $>$ Qingshui River valley basin area, tributary area $>$ trunk stream area, upstream area $>$ middle reaches area $>$ downstream area of Qingshui River. This is relatively consistent with the spatial distribution of fluoride ion content in Qingshui River groundwater.
\end{abstract}

Keywords Endemic fluorosis $\cdot$ Arid area $\cdot$ High-fluorine water $\cdot$ Spatial distribution

\section{Introduction}

Fluorosis is a widespread endemic disease in China. As early as 1980, a general survey identified cases of fluorosis in more than 7100 villages, distributed over 1000 counties in China. It

Responsible editor: Philippe Garrigues

Mingji Li

li_mj@nxu.edu.cn

Hong Miao

miaohong1101@126.com

1 Resource and Environment Department, Ningxia University, Yinchun 750021, China

2 Western Ecology Research Center, Ningxia University, Yinchun 750021, China is estimated that there are currently over 21 million patients with dental fluorosis and over 10 million patients with skeletal fluorosis in China (DDCMH, China). The cause of these conditions is in most cases excessive uptake of fluorine via drinking water. Low but regular uptake of fluorine is beneficial for dental health, but when present concentrations are above $1 \mathrm{mg} / \mathrm{L}$ in drinking water, it can lead to dental fluorosis, and at concentrations beyond $3 \mathrm{mg} / \mathrm{L}$, the incidence of dental fluorosis will reach $100 \%$ for exposed individuals. The World Health Organization (WHO) advises a maximum allowable fluorine concentration of $1.5 \mathrm{mg} / \mathrm{L}$ in drinking water (Fawell 2006).

The occurrence of dental fluorosis is further related to local temperatures (related to increased water consumption in warmer climates), intake of calcium and phosphorus, and individual differences. An imbalance of vitamins $\mathrm{A}$ and $\mathrm{D}$, and $\mathrm{Ca}$ and $\mathrm{P}$ uptake can increase the dental damage associated 
with fluorine (Li 2004). Drinking water guidelines set by the Chinese government require the fluorine concentration in $\mathrm{mu}-$ nicipal drinking water to remain within the range of $0.5-$ $1.0 \mathrm{mg} / \mathrm{L}$. In recent years, efforts have been made to mitigate adverse effects of endemic fluorosis in high-fluorine areas, through improving water quality and, when needed, by relocating communities through the project of eco-migration conducted by the government; from the 1980 s to the end of 2016, the ecological migration project in Ningxia, led by the local government, has relocated more than 1 million people from the contiguous poverty-stricken areas of the Liupanshan Mountains to the areas along the Yellow River. The Ningxia ecological migration project is a process of population spatial reconstruction under the response of ecological pressure in the ecologically fragile area and the extremely poor area of Liupanshan Mountains.

Owing to the relatively high prevalence of endemic fluorosis in China, the fluorine distribution, origin, and other factors responsible for the disease have frequently been investigated. Liu and colleagues demonstrated in 1980 that endemic fluorosis was mainly concentrated in 3 regions within China: an east-to-west belt in the northern deserts, a region along the northern coast, and a region in the south, with a number of localized smaller areas elsewhere. Groundwater with a high fluorine content was correlating with geological conditions, soil lithology, and hydrochemical characteristics in Northern China (Liu et al. 1980), while in Inner Mongolia it was mainly detected in the alluvial-pluvial plain at the mountain front (Feng et al. 2015), suggesting an origin from fluorinecontaining minerals and rocks in the surrounding mountains. A correlation with soils and groundwater rich in sodium carbonate was identified (He et al. 2010). Low $\mathrm{Ca}^{2+}$ concentrations further favor the formation of high-fluorine groundwater, as is a slight alkalinity (Mao et al. 2016). This was demonstrated in the plain and mountain front of Inner Mongolia, where $\mathrm{pH}$ and concentrations of $\mathrm{HCO}_{3}{ }^{-}, \delta^{18} \mathrm{O}, \delta \mathrm{D}$, and total dissolved solids (TDS) were all higher in high-fluorine groundwater collected in the plain than those in the mountain front (Li et al. 2018). Similar findings were reported from inland river basins in Yanchi, Ningxia (Wu et al. 2018). Piper trilinear diagrams were used to indicate the groundwater facies are basically identical in different seasons, and hydrochemical facies are of the $\mathrm{Na}-\mathrm{Cl}$ type and $\mathrm{Na}-\mathrm{SO}_{4}-\mathrm{Cl}$ type, while Gibbs diagrams illustrated that groundwaters reside in evaporation-dominant areas, highlighting the crucial role of evaporation in the chemistry of groundwater $(\mathrm{Wu}$ et al. 2018).

Current investigations of high-fluorine water and its consequences to human health mainly focus on (a) the occurrence, classification, and pathogenesis of endemic fluorosis; (b) sources, distribution, and formation of high-fluorine water; and (c) the hydrogeochemistry of fluorine. However, the correlation of river water high in fluorine is less well investigated, while the collective influence of river water, groundwater, and rainfall in China remains to be assessed. This is particularly important in the high-fluorine region in northwestern China, where a loess plateau is rich in carbonate with an alkaline $\mathrm{pH}$ (Liu et al. 1966), and fluorine-bearing minerals such as mica, amphiboles, tourmaline, and apatite are abundant. Through rainfall, groundwater leaching, circulation, and exchange between groundwater and surface water, fluorine can be released from these minerals to be introduced into surface water including river water.

Our research studies the coupling relationship between the fluorine content and the incidence of endemic fluorosis in the Qingshui river basin. And we aim to investigate groundwater and surface water are the main reasons that affecting the health of residents in the basin, and encourge the local government of Ningxia to carry out water reform projects and migration projects.

\section{Material and methods}

\section{Study area}

Ningxia is a land-locked province in the north of China (Fig. 1, right). The Qingshui River flows through Ningxia and its basin (shaded gray in the figure) covers 4 counties (Xiji, Tongxin, Haiyuan, and part of Zhongning County) and 3 districts (Yuanzhou, Hongsibu, and Shapotou Districts)that together account for $49.98 \%$ of the land surface of Ningxia. Among them, Xiji, Tongxin, and Haiyuan Counties and Yuanzhou District are part of a poverty-stricken area of the Liupanshan Mountains designated by the Chinese government to receive special attention to mitigate poverty-related problems including improvement of drinking water quality. The eastern and south-central parts of Ningxia see a high incidence of endemic fluorosis and goiter. The local climate is arid with little rainfall and water supply is limited. In the south-central area, the annual rainfall is about $300 \mathrm{~mm}$, mostly occurring during the wet season from July to October, whereas annual evaporation exceeds $2300 \mathrm{~mm}$. Local populations often use untreated spring and well water as sources for drinking water despite its poor quality. The Qingshui River system is the only first tributary of the Yellow River in central and southern Ningxia. Qingshui River basin is the main area where the Qingshui River and the shallow groundwater in the basin resupply and transform each other.

The local geography surrounding the river basin is characterized by mountains with thick strata deposited during the Jurassic, Cretaceous, and Tertiary Periods. These strata are rich in water-soluble salts such as gypsum $\left(\mathrm{CaSO}_{4}\right)$ and mirabilite $\left(\mathrm{Na}_{2} \mathrm{SO}_{4}\right)$ and relatively rich in fluoride. As a result, groundwater is often brackish with a mineralization degree of 


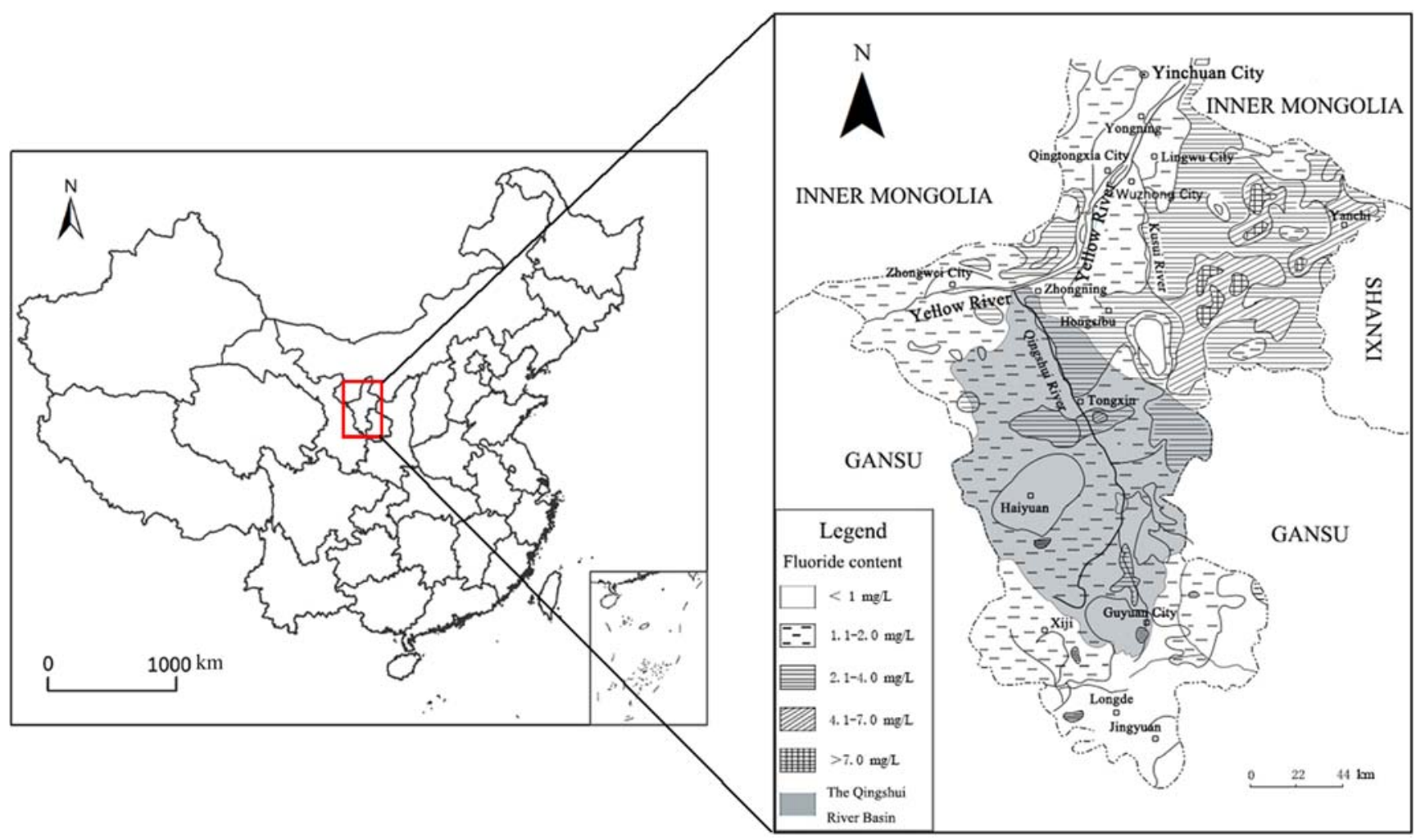

Fig. 1 Map of location and fluorine concentrations

$30-150 \mathrm{~g} / \mathrm{L}$ and a fluorine content of around $1.29 \mathrm{mg} / \mathrm{L}$ on average (Fig. 1, left).

Shallow groundwater is the main source of drinking water for a long time in Qingshui River basin, where the underground water is also the major source of catchment for Qingshui River. Especially in the dry season, almost all of the river is supplied by the groundwater. Therefore, the Qingshui River water can be used as the sample to clarify the influence of groundwater on endemic fluorosis in the river valley basin.

\section{Epidemiological data on fluorosis}

The historical data on prevalence of fluorosis used here were obtained from Ningxia Center for Diseases Prevention and Control. These included the raw data from a survey performed in 1981, data from a survey from 2008 and 2009, an annual assessment on endemic fluorosis from 2010, and monitoring data of endemic fluorosis from the years 2015 and 2017.

From 2009 to 2014, skeletal fluorosis of endemic fluorosis data were collected from 27 sample villages in 9 regions, i.e., Lingwu City, Litong District, Tongxin County, Yanchi County, Qingtongxia City, Pengyang County, Xiji County, Haiyuan County, and Shapotou District. All these data were used in combination to investigate the variation in endemic fluorosis over a period of 40 years.

\section{Local river water sampling}

Water samples were collected from the Qingshui River (main stream and tributary waters) and groundwater in 2018-2019. For this, 20 sampling points were evenly set up, and sampling was conducted three times at each point during April, July, and November.

\section{Analytical methods}

The $\mathrm{pH}$, TDS, and electrical conductivity (EC) were determined on-site using a portable $\mathrm{pH}$ meter and a conductivity meter, respectively. Water samples were preserved in 5-L precleaned and sterilized polyethylene bottles, then sealed and transported to the laboratory within $24 \mathrm{~h}$, where they were stored at $4{ }^{\circ} \mathrm{C}$ and used within $48 \mathrm{~h}$.

Soluble metals of the water samples such as $\mathrm{Na}^{+}, \mathrm{K}^{+}, \mathrm{Ca}^{2+}$, and $\mathrm{Mg}^{2+}$ were analyzed by inductively coupled plasma mass spectrometry (ICP-MS, Agilent 7500a, Santa Clara, CA, USA). Anions including $\mathrm{NO}_{3-}, \mathrm{NO}_{2-}, \mathrm{SO}_{4-}, \mathrm{CI}-$, and $\mathrm{F}-$ were detected by ion chromatography (Metrohm 761/813, Switzerland). The accuracy and precision of all indexes of groundwater sample analysis are in line with the quality requirements, the relative error of anion and Yang balance is less than $5 \%$, and the qualified rate of sample test is $100 \%$.

The color scale shows the number of affected villages with fewer than 200 affected villages are considered as low 
endemic; areas with between 200 and 400 affected villages, medium endemic; and areas with over 400 affected villages, high endemic.

\section{Results}

\section{Analysis of epidemiological data of endemic fluorosis (1981-2017)}

Endemic fluorosis in the investigated area was first described in the survey of 1981. This covered a population of 4.87 million in 20 cities/counties of Ningxia, in which all but one fluorosis was endemic to various degrees (the exception was Jingyuan County). In that year, endemic fluorosis was reported for 539 villages in the region. The situation worsened over time. In 2010, there were 3449 villages with endemic fluorosis; in 2015, 3400 villages were affected. The number of affected villages decreased to 3269 by the end of 2017, while several small villages merged by administrative changes, and the endemic fluorosis problem was still not improved. The worst affected locations were Yuanzhou District, Xiji County, Tongxin County, and Zhongning County, as these contained the most villages reporting endemic fluorosis. A distribution map of affected villages is shown in Fig. 2. The total affected area could be divided into low-endemic areas where fewer than 200 villages per $1000 \mathrm{~km}^{2}$ were affected, a medium-endemic area with between 200 and 400 villages affected, and a highly affected area where over 400 villages per $1000 \mathrm{~km}^{2}$ reported fluorosis cases.

Not only the number of affected villages but also the affected population increased significantly from 1981 to 2001, as summarized in Table 1.

From 1981 to 2001, the population suffering from fluorosis increased significantly in Ningxia. The fluorosis population was 640,454 in 1981 (355,072 in 278 slight fluorosis areas; 181,909 in 161 medium fluorosis areas; 103,473 in 100 serious fluorosis areas). In 2010, there were 1950 slight fluorosis areas, 1154 medium fluorosis areas, and 1345 serious fluorosis areas. Compared with the data in 1981, the number of serious, medium, and slight fluorosis areas increased by $71 \%, 86 \%$, and nearly $86 \%$, respectively.

During the period from 1981 to 2010 , there was an increase of $25.5 \%$ of the patients of high-endemic regions, while patients in low- and medium-endemic regions increased by $49 \%$ and $126 \%$. The affected patients nearly doubled over the investigated period (Qingshui River basin), resulting in 1.1 million people living in affected areas by 2017 (16.43\% of the total population of Ningxia).

Of the affected Ninxia population, 62.3\% lived in Qingshui River basin. The most severely affected regions were the Yuanzhou District and Xiji County in the Qingshui River
Fig. 2 Distribution of villages reporting fluorosis cases in Ningxia, China, in 2017

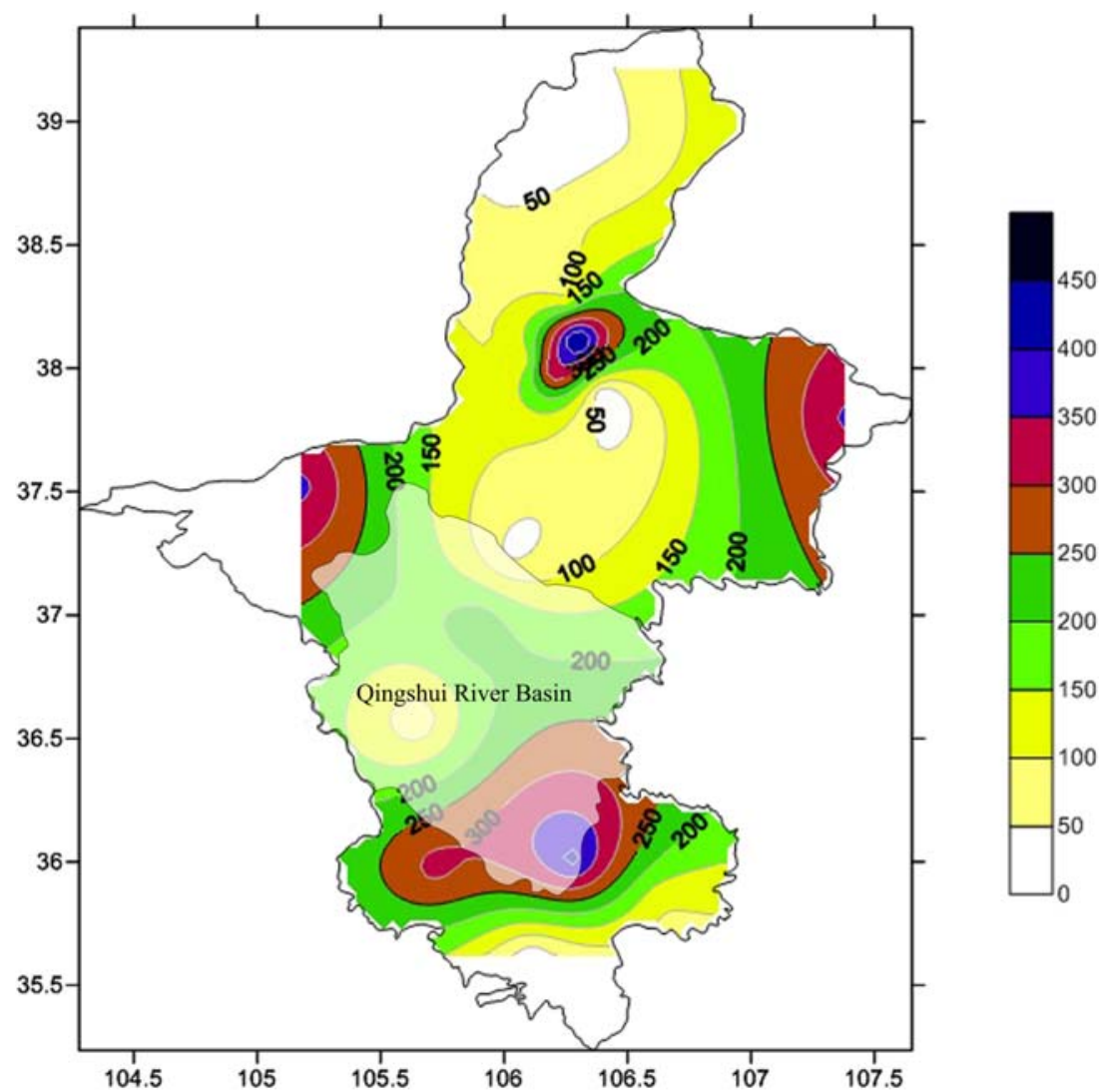


Table 1 Historical trends in populations affected by fluorosis in Ningxia, China

\begin{tabular}{llllll}
\hline \multirow{2}{*}{ Year } & Total population & \multicolumn{2}{l}{ Cases of fluorosis and number of villages affected (in brackets) } \\
\cline { 3 - 6 } & & Affected population & Low-endemic regions & Medium-endemic regions & High-endemic regions \\
\hline 1981 & 4.87 million & $640,454(n=539)$ & $355,072(n=278)$ & $181,909(n=161)$ & $103,473(n=100)$ \\
2010 & 6.33 million & 1.07 million $(n=3449)$ & $529,532(n=1950)$ & $411,749(n=1154)$ & $129,913(n=1345)$ \\
2017 & 6.70 million & 1.10 million $(n=3269)$ & - & - & - \\
\hline
\end{tabular}

After 2015, the local fluorosis in Ningxia can no longer be classified as mild, moderate, and severe

basin. The spatial distribution of affected populations is illustrated in Fig. 3 (number of villages).

\section{Spatial distribution of skeletal fluorosis in Ningxia}

Since 2009, Ningxia Disease Control and Prevention Center commenced to monitoring endemic fluorosis associated with drinking water in 27 natural villages of fluorosis areas in 9 counties/cities/districts (Lingwu City, Litong District, Tongxin County, Yanchi County, Qingtongxia City, Pengyang County, Xiji County, Haiyuan County, and Shapotou District). Skeletal fluorosis was monitored every 5 years. In 2009 and in 2014, 27 villages highly affected by fluorosis were monitored specifically and data on skeletal fluorosis are available. This condition was detected in 550 out of 1384 patients in 2009 (39.74\%). A majority of these cases were detected in low-endemic areas (474 cases, $35.84 \%)$, while $44(3.18 \%)$ were from medium-endemic and $32(2.31 \%)$ from high-endemic areas. In 2014, 519 skeletal fluorosis cases out of 1398 were identified in the same villages (37.12\%), indicating the situation had only slightly improved. However, fewer cases were now present in medium- and highendemic areas (496 (35.5\%) in low-, 18 (1.29\%) in medium-, and $5(0.36 \%)$ in high-endemic areas).

In 2009 and 2014, the number of cases of fluorosis detected in Qingshuihe River basin accounted for $38.56 \%$ and $35.23 \%$ respectively of Ningxia.

\section{Spatial distribution of dental fluorosis in children}

Dental fluorosis was common in Ningxia. Specific data on dental fluorosis in children (aged 8 to 12 years) were available
Fig. 3 Distribution of fluorosis populations in Ningxia in 2017

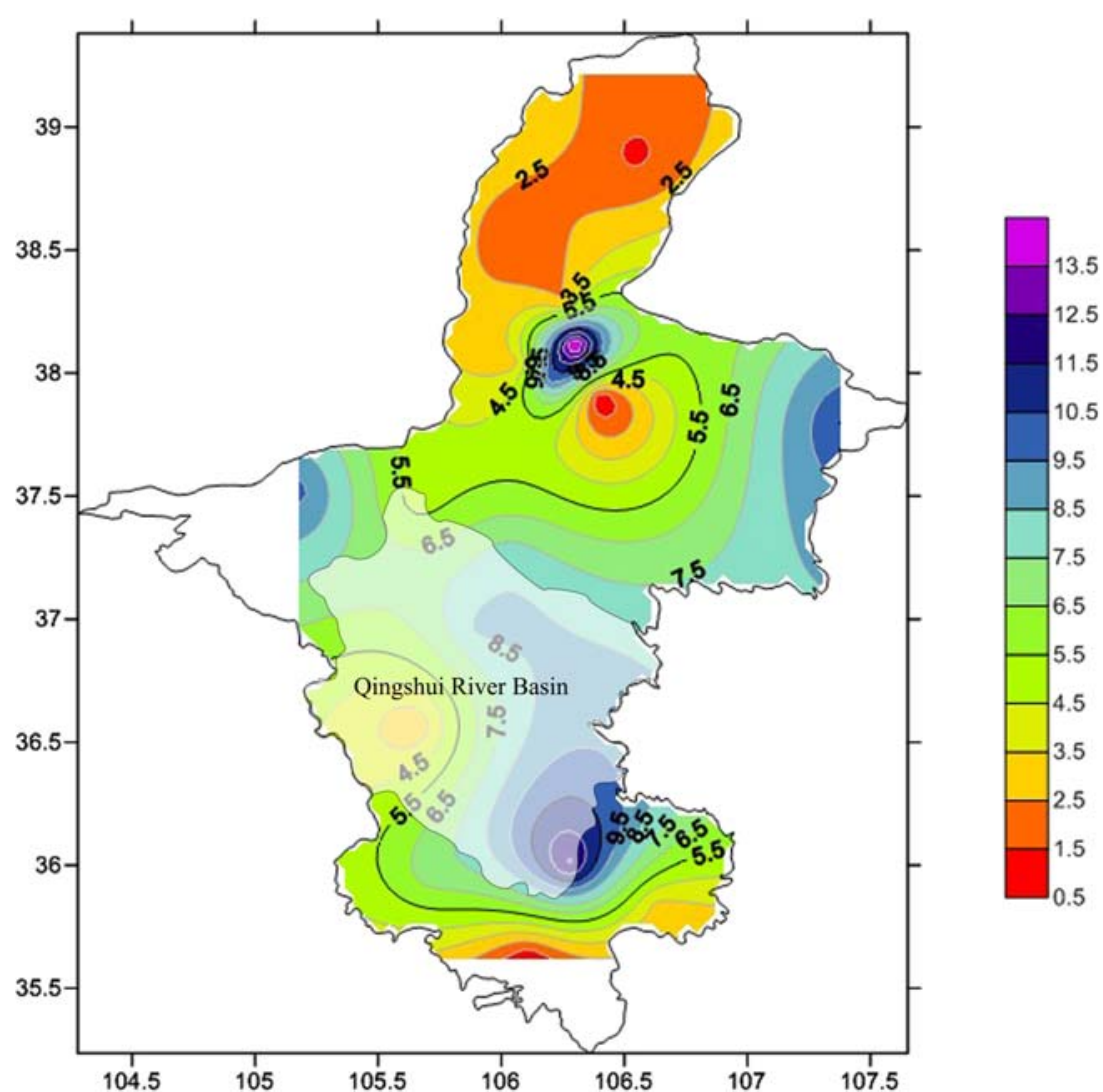


for 2015 (this statistics have only been officially conducted in 2015). Dental fluorosis is particularly serious in children in Qingshui River basin, and especially serious in Xiji County, where 1616 of children were affected, followed by Yuanzhou District (758) and Lingwu City (602). These absolute numbers gave an incidence of 459.74 (Xiji County) and 331.15 (Yanchi County) per 1,00,000, respectively, which was much higher than the average incidence for the whole of Ningxia of dental fluorosis in children (96.58 per 100,000) (Table 2). A spatial distribution of dental fluorosis in children (2015) is shown in Fig. 4.

\section{Geological foundation for high concentrations of fluoride}

The local geology of the Qingshui River basin is rich in fluorine-bearing minerals. The Madongshan and Naijiahe Formations of the Lower Cretaceous Series in Qingshuiying and Hongliugou contain 193-932 mg/kg fluorine, and the Ganhegou Formations of the Neogene Miocene Series contain $629 \mathrm{mg} / \mathrm{kg}$ on average (Wu et al. 1999), which is higher than the average fluoride content $(420 \mathrm{mg} / \mathrm{kg})$ in rocks in China. When groundwater flows through fluoride-bearing rocks, the fluoride can dissolve, resulting in higher fluoride concentrations in groundwater.

Runoff water greatly fluctuates annually in the Qingshui River basin. The maximum annual runoff is $2.08 \times 108 \mathrm{~m}^{3}$, approximately triple that of the minimum $\left(0.67 \times 108 \mathrm{~m}^{3}\right)$. The coefficient of variation $(\mathrm{Cv})$ is 0.28 . During the dry season, sources of river runoff mainly rely on the recharge of shallow groundwater, e.g., water in fissured bedrock and pore water in loose sediments. During the wet season, the flowing river has high water levels so the recharge sources are mainly from rainfall runoff. The main arm of the Qingshui River is mostly recharged through rainfall, tributary import, and lateral recharge of groundwater from both sides. Thereby, it is significantly impacted by geomorphology, climate, and local hydrogeological conditions.
The fluorine concentration of Qingshui River surface water was determined, both for the main stream and for the tributary waters. The fluorine concentration of the main stream varied from 0.18 to $2.85 \mathrm{mg} / \mathrm{L}$ with $1.14 \mathrm{mg} / \mathrm{L}$ on average, and that of the tributary from 0.44 to $3.86 \mathrm{mg} / \mathrm{L}$ with $0.91 \mathrm{mg} / \mathrm{L}$ on average. The highest values for both waters were obtained in April during the dry season, and the lowest in July during the wet season (Fig. 5). Values obtained in November were within these extremes. This reveals the significant role of surface runoff in regulating fluorine concentration in the surface water. During both the dry and wet seasons, the average fluorine concentration of main stream water was lower than that of tributary water, implying that the tributary water contributed significantly to the fluorine content of the main stream water. The higher concentration of fluorine in tributary water during the dry and wet periods was most likely caused by the confluence of groundwater containing high concentrations of fluorine. In November (normal season), the average fluorine concentration of main stream water was higher than that of tributary, implying that the import of tributary into main stream waters no longer exerted an additive effect on fluorine concentration. During the dry season, rivers are mainly replenished with groundwater, which in this case is rich in fluorine due to leaching from fluoride-bearing rocks. During the wet season, the rivers are mainly replenished via rainfall, thereby diluting the overall fluoride concentration. Nevertheless, the tributary waters produced higher concentrations, most likely because the main river was fed by upstream alluvial sources low in fluorine. During the normal season, the latter effect seemed to be weaker, resulting in higher fluorine concentrations in the main river.

Other anions $\left(\mathrm{Cl}^{-}, \mathrm{SO}_{4}{ }^{2-}, \mathrm{CO}_{3}{ }^{2-}\right.$, and $\left.\mathrm{HCO}_{3}{ }^{-}\right)$were also assessed in the water samples, as were the cations $\mathrm{Na}^{+}, \mathrm{K}^{+}$, $\mathrm{Ca}^{2+}$, and $\mathrm{Mg}^{+}$. A Piper trilinear diagram was constructed (Fig. 6). The water from the Qingshui River contains $\mathrm{Na}^{+}$as the dominant cation, and $\mathrm{Cl}^{-}$and $\mathrm{SO}_{4}{ }^{2-}$ as predominant anions. The hydrochemical facies is of $\mathrm{Na}-\mathrm{K}-\mathrm{SO}_{4}$ type, indicating that the river is influenced by $\mathrm{Cl}^{-}$and $\mathrm{SO}_{4}{ }^{2-}$. This is consistent with the presence of halite and gypsum sediments

Table 2 Statistics of dental fluorosis in children distribution in Ningxia based on grade of dental fluorosis population and incidence

\begin{tabular}{lll}
\hline Grade of fluorosis population & Grade of fluorosis incidence (per 1,000,000) & Distribution \\
\hline Low $(<17)$ & Low $(<8.72)$ & Yongning County, Shatoupo District, and Longde County \\
$\begin{array}{l}\text { Relatively low } \\
(17-83)\end{array}$ & $\begin{array}{l}\text { Relatively low } \\
(8.72-28.48) \\
\text { Medium (83-284) }\end{array}$ & $\begin{array}{c}\text { Xingqing District, Huinong District, Pingluo County, } \\
\text { and Haiyuan County }\end{array}$ \\
Relatively high (284-505) & $\begin{array}{l}\text { Relatively high } \\
(81.16-182.39)\end{array}$ & $\begin{array}{c}\text { Hongibu District, Pengyang County, Qingtongxia City, } \\
\text { and Tongin County }\end{array}$ \\
High $(\geq 505)$ & High $(\geq 182.39)$ & Dawukou District, Zhongning County, and Litong District \\
& & Xiji County, Yanchi County, Lingwu City, and \\
\end{tabular}



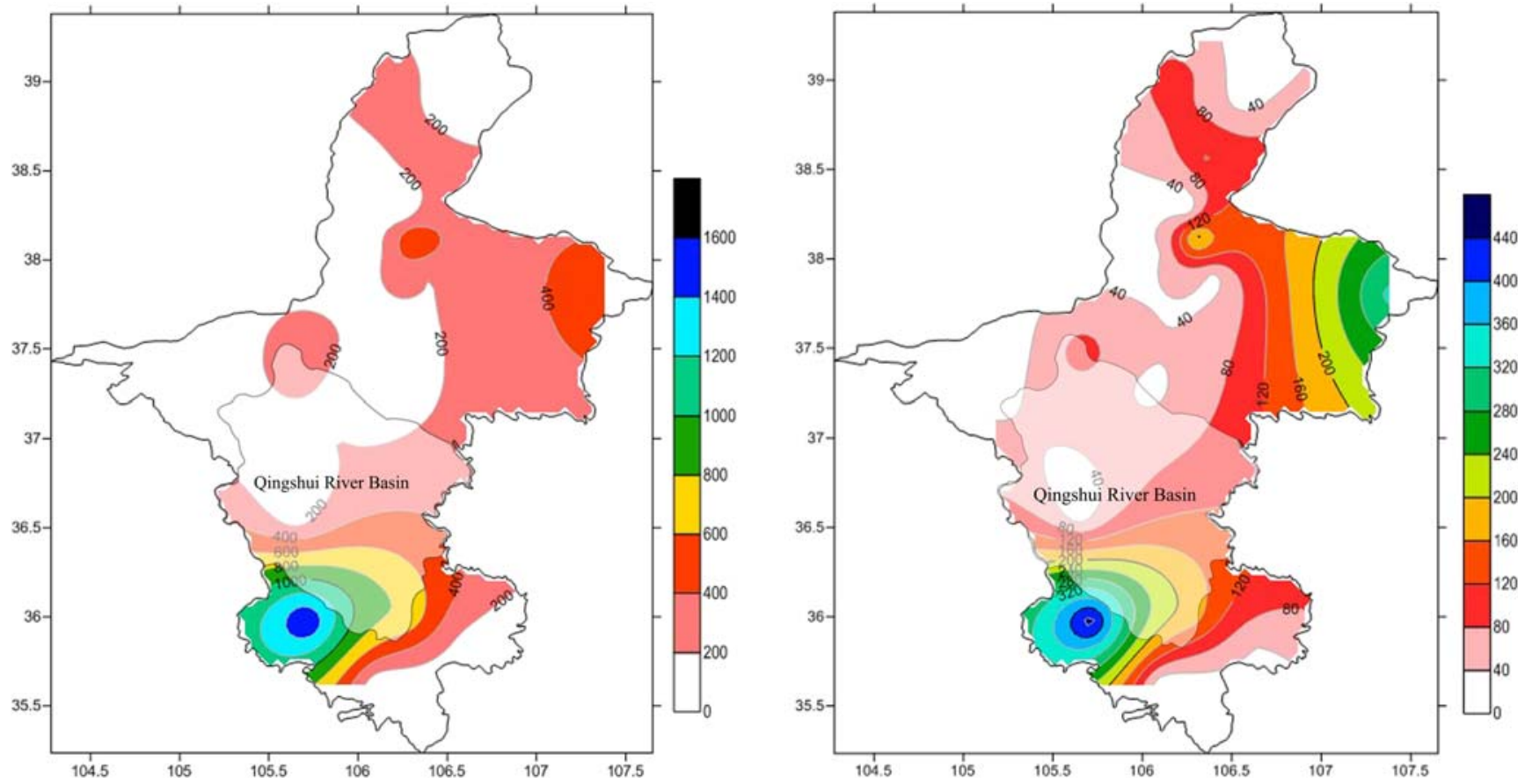

Fig. 4 a Spatial distribution of dental fluorosis cases in children in Ningxia. b Spatial distribution of its grade incidence

in the basin. Through the action of hydrodynamic forces and leaching, these sediments can release large amounts of $\mathrm{Na}^{+}$, $\mathrm{Cl}^{-}$, and $\mathrm{SO}_{4}{ }^{2-}$, which subsequently enter surface waters including the river.

\section{Discussion}

The fluorine content of groundwater has been widely investigated during past decades, in locations around the globe. Robertson investigated the distribution, migration, and enrichment of fluorine in groundwater in alluvial-pluvial basins of Arizona, USA (Robertson 1984), which revealed that the fluorine in groundwater largely originated from ion exchange and fluorine-bearing mineral dissolution. Lahermo described that the fluorine distribution in groundwater in Finland and East Africa was associated with the distribution of fluorite, granite, gneiss, and crystalline granite (Lahermo et al. 1991). Indeed, the dissolution of fluorine-bearing minerals is commonly regarded as the source of fluorine in groundwater (McCaffrey and Willis 1993). This dissolved fluorine is then transported to enter groundwater in processes dictated by geological structural units such as strata of fault zones (Apambire et al. 1997). External factors, such as hydrochemical facies, geomorphology, and microtopography, and human activities all contribute to groundwater reaching high concentrations of fluorine (Conrad et al. 1999; Kundu 2001; Levy and Schramke 1999). Carrillo-Rivera et al. explored the distribution of high-fluorine groundwater in the San Luis Potosi basin, Mexico, and demonstrated that there high-fluorine groundwater was dependent on hydrogeological conditions, temperature, and fluoride concentration in the vadose zone (Carrillo-Rivera et al. 2002). Rukah et al. conducted a geochemical assessment on fluoride contamination of groundwater in North Jordan (Rukah and Alsokhny 2004), who reported a positive correlation with $\mathrm{pH}$ and bicarbonate concentration. Alvarez et al. clarified the source of fluorine through analyzing the relation between aquifer sediments and ionized water in Patagonia, Argentina (Del Pilar Alvarez and Carol 2019). The similar contour and the spatial distribution of fluoride in the phreatic aquifer were reported in Toketo, Inner Mongolia Autonomous Region, China, which was mainly controlled by groundwater flow. There, a high-fluorine area $(>5 \mathrm{mg} / \mathrm{L}$ in groundwater) was located in a discharge area which was shallowly buried and strongly exposed to evaporation (Feng et al. 2016). In addition, the relation between groundwater and phreatic aquifer is also similar to that in Nagar Parkar area, Sindh, Pakistan, i.e., the concentration of fluorine in the upstream is much higher than that in midstream/downstream (Rafique 2009).

Our results are consistent with these reported findings, whereas the sources of fluorine in groundwater are multiple and vary locally as they are dependent on different climate conditions, local geology, and other local settings. From the data analyzed here, we conclude that fluorine in the Qingshui River basin dominantly originates from chemical weathering of fluorine-bearing minerals. This leaching contaminates groundwater that feeds into surface waters, where evaporation leads to higher concentrations and rainfall to diluted concentrations. Thus, the overall concentrations are associated with 

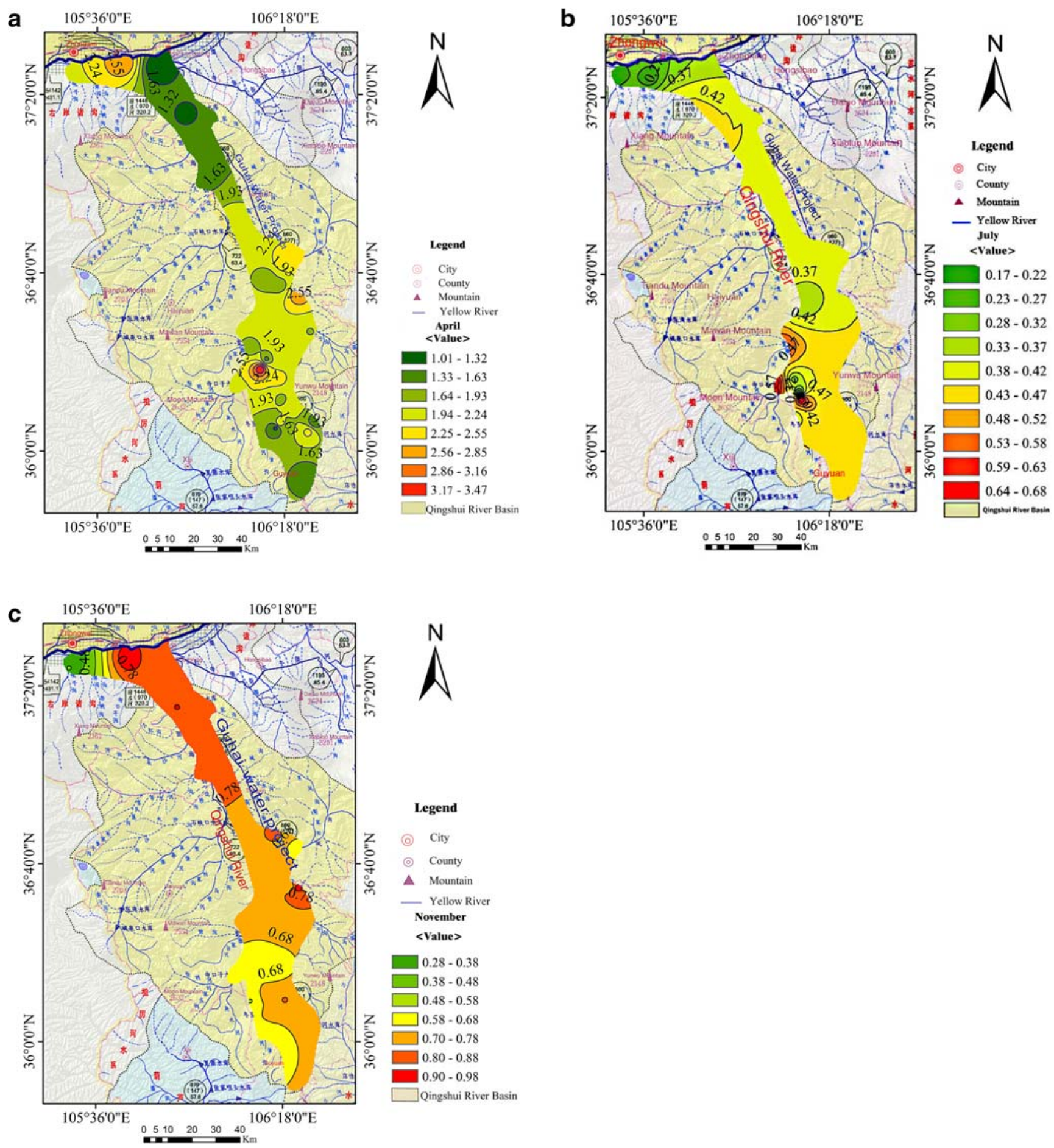

Fig. 5 Spatial distribution of surface water fluorine concentrations in Qingshui River basin in April (a), in July (b), and in November (c)

geological features of these arid and semi-arid areas as well as the basin structure, hydrochemical facies of the groundwater, and local climate conditions. Due to less precipitation than evaporation, surface waters are being enriched in fluoride. High concentrations of fluorine in the Qingshui River are the results of water-rock interaction and evaporation. The latter increases TDS and fluoride concentrations; intensified ionic strength as a result of Qingshui River further enhances the solubility of fluoride.

The main source for drinking water in the investigated region is well water. Although well water was not directly investigated, the high fluorine concentrations detected in river water suggest that both groundwater and well water will also have a high fluorine content. The consumption of such 
Fig. 6 A Piper trilinear diagram of water samples of the Qingshui River basin

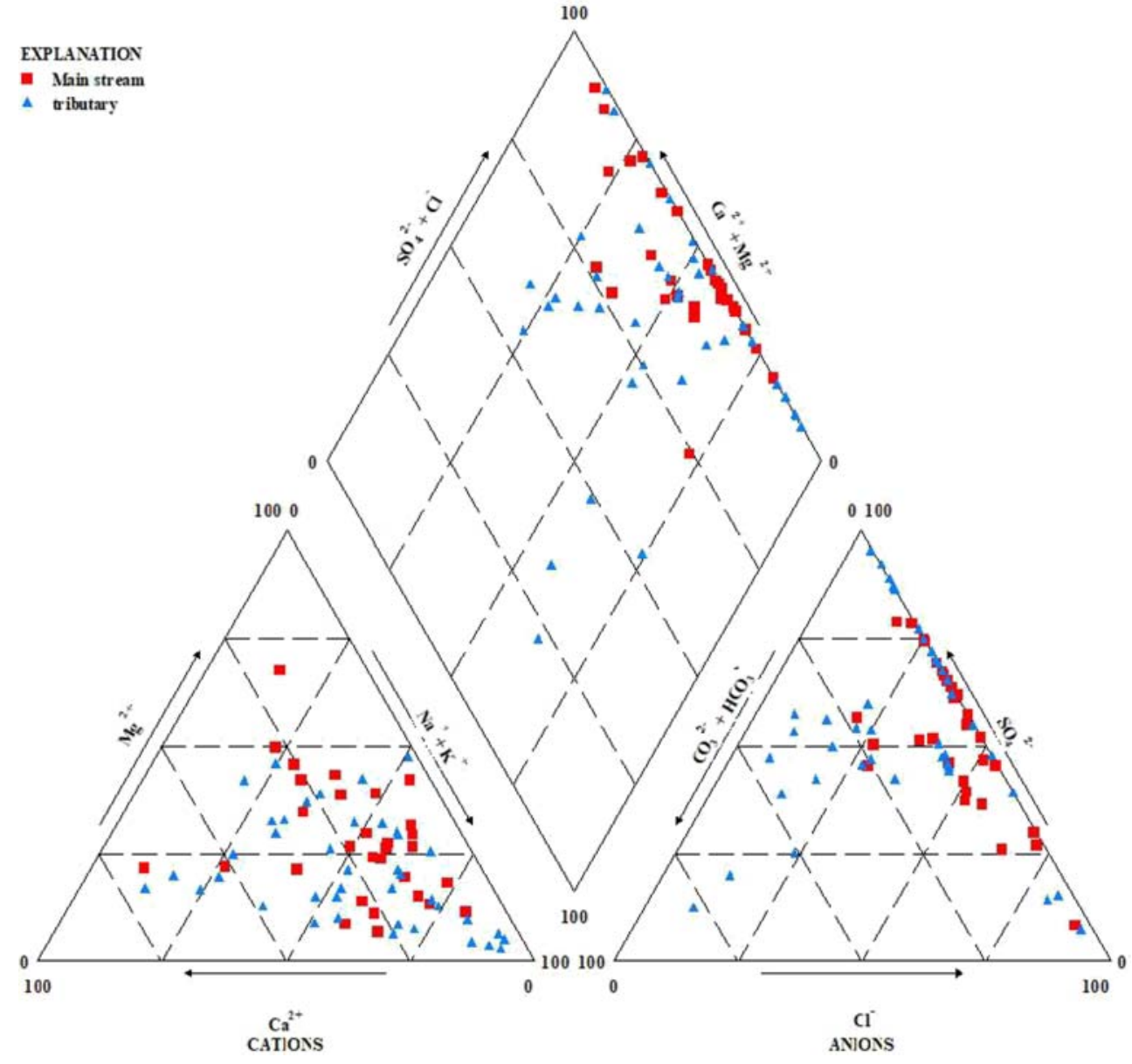

contaminated drinking water is the most likely cause for the high incidence of fluorosis reported for this area. The data collected since the 1980s identified multiple regions with a high incidence of endemic fluorosis, especially in southcentral Ningxia. Fluorosis cases concentrate in two villages located in the upstream region of the Qingshui River within the Yuanzhou District and in Xiji County along one of its tributary arms. The fluorosis incidence decreases further downstream of the Qingshui River. The spatial distribution of villages and population of endemic fluorosis present the following features: headwater $>$ river valley basin; mainstream $>$ tributary; upstream $>$ midstream $>$ downstream. These distribution features are basically in agreement with the contour of fluoride in the groundwater and the spatial distribution of fluorine concentration in the phreatic aquifer in Qingshui River basin.

\section{Conclusion}

At present, 2019-nCoV acute respiratory disease is in the process of comprehensive prevention and control in China. The national disease control system is the most important backbone in the prevention and control of this acute infectious disease. Similarly, in the prevention and control of endemic diseases, the CDC system also plays an extremely important role. The prevention and control of endemic fluorosis by the Ningxia disease control center and local government has been implemented since the beginning of the 1950s. The problem of endemic fluorosis in the south-central region of Ningxia has been solved through universal salt iodization, medical treatment, and other measures. After the 1980s, through the general survey of endemic diseases and dynamic monitoring of endemic diseases control departments at all levels, a comprehensive investigation and study on endemic fluorosis in Ningxia was carried out. On this basis, local disease control departments try to change the situation of high incidence of endemic fluorosis in South-Central region of Ningxia through a series of comprehensive engineering projects. The local government has relocated about 1 million people of south-central region of Ningxia by ecological migration project to the plain and high platform area along Yellow River, the Yellow River water as the main drinking water source, which greatly reduced the incidence rate of endemic fluorosis. At the same time, the local government of Ningxia diverted the Yellow River water to Tongxin County, Haiyuan County, Hongsibu 
District, Yuanzhou District, and other places in the southcentral region of Ningxia through the water diversion project, which changed the habit of drinking shallow groundwater and Qingshui River water in these areas, and solved the drinking water problem of most of the population in the areas with a high incidence of endemic fluorosis. From 2000 to 2017, the total number of endemic villages was showing a downward trend, especially the more serious endemic fluorosis such as skeletal fluorosis and the number of people were gradually decreasing. However, due to the relatively scattered administrative villages in the south-central region of Ningxia, there are still some villages taking shallow groundwater as drinking water. The local governments need to build large and medium-sized reservoirs as the primary drinking water source to further solve the drinking water problems of these villages, so as to completely eliminate endemic fluorosis in the region.

The coupling relationship between rivers which contain high concentration of fluoride ions formed by the convergence of groundwater and precipitation and endemic fluorosis of subregion in northwest China is less studied at home and abroad. Based on the historical 40 years data on endemic fluorosis and the spatial distribution and formation mechanism of high concentrations of fluoride in Qingshui River basin in Ningxia, this study concludes that there is a close coupling relationship in south-central Ningxia between the endemic areas of endemic fluorosis and the spatial and temporal distribution of excess fluorine water of Qingshui River basin. This relationship indicates that fluoride in the groundwater of the Qingshui River basin has long been affected by geological structures, low-lying and closed terrain, and drought. Qingshui River and shallow groundwater in the basin have a strong process of mutual resupply and transformation. And we consider the phreatic aquifer of the basin as the prominent contributor for endemic fluorosis because shallow groundwater is the main source of drinking water for a long time in this region. The temporal and spatial distribution of regional endemic fluorosis with long time series and its corresponding relation with water fluoride content in watershed were discussed. Then, we preliminarily concluded that the upper part of the basin is the high-incidence areas, and the middle and lower reaches are the middle and lower levels. The spatial distribution of villages and population of endemic fluorosis presents following features: headwater $>$ river valley basin; mainstream $>$ tributary; upstream $>$ midstream $>$ downstream. These distribution features are basically in agreement with the contour of fluoride in the groundwater and the spatial distribution of fluorine concentration in the phreatic aquifer in Qingshui River basin. This conclusion confirmed the source, settlement, and the change process of fluoride concentration in precipitation catchment in different seasons, and its influence on the endemic fluorosis areas. The results are of great significance in scientific research. However, the monitoring time series of the basin is relatively short in the research process, and there is still insufficient correspondence with the historical data of the disease, so further follow-up research is necessary. At the same time, this study is also of great practical significance for local governments to control endemic fluorosis. We believe that the Ningxia government should continue to carry out water improvement measures through the water diversion project of the Yellow River, so as to govern the endemic fluorosis in the Qingshui River basin from the source. Furthermore, implement "targeted poverty alleviation" and ecological migration poverty alleviation projects, and move the poor people in the uninhabitable endemic fluorosis areas to the Yellow River-irrigated oasis areas such as the plain and the high platform, with the Yellow River water as the main drinking water source, so as to thoroughly solve the situation of high incidence of endemic fluorosis in the areas.

Funding information This work was supported by the National Natural Science Foundation of China (Grant Nos. 41961028, 41561037, and 41461119), and the Environmental Research Projects of Ecoenvironment Department of Ningxia Hui Autonomous Region (2018-07).

\section{References}

Apambire WB, Boyle OR, Michel FA (1997) Geo-chemistry genesis and health implications of fluoriferous ground waters in the upper regions of Ghana. Environ Geol 33(1):13-18

Carrillo-Rivera JJ, Cardona A, Edmunds WM (2002) Use of abstraction regime and knowledge of hydrogeological conditions to control high-fluoride concentration in abstracted groundwater: San Luis Potosi basin, Mexico. J Hydrol 261(1-4):24-47

Conrad PG, Carey DL, Webb JS et al (1999) Ground-water Quality in Kentucky: fluoride. Inform Circ 1 Series XII:1-3

Del Pilar Alvarez M, Carol E (2019) Geochemical occurrence of arsenic, vanadium and fluoride in groundwater of Patagonia, Argentina: sources and mobilization processes. J S Am Earth Sci 89:1-9

Fawell JK (2006) Fluoride in drinking-water[M]. World Health Organization, Geneva

Feng C-E, Gao C-R, Wang J-T et al (2015) Distribution and causes of high-iron and high-fluoride shallow groundwater in the Hetao Plain of Inner Mongolia. Acta Geosci Sin 36(1):67-76 (in Chinese)

Feng H-B, Dong S-G, Shi X-L et al (2016) The spatial distribution and its formed mechanism of fluoride in the unconfined and confined groundwater of Tuoketuo County, Inner Mongolia. Geoscience 30(3):672-679 (in Chinese)

He J, Zhang F-C, Han S-B et al (2010) The distribution and genetic types of high-fluoride groundwater in northern China. Geol China 37(3): 621-626 (in Chinese)

Kundu N (2001) Geochemical appraisal of fluoride contamination of groundwater in the Nayagarh District of Orissa India. Cases Solutions 15:18-21

Lahermo P, Sandström H, Malisa E (1991) The occurrence and geochemistry of fluorides in natural waters in Finland and East Africa with reference to their geomedical implications. J Geochem Explor 41(12):65-79

Levy DB, Schramke JA (1999) The shallow groundwater chemistry of arsenic, fluoride, and major elements: Eastern Owens Lake, California. Appl Geochem 14:53-65

Li G-S(2004) Pathogenesis of endemic fluorosis. Science Presss, BeiJing, pp 4-25 Chief Editor; (in Chinese) 
Li D, Gao X, Wang Y et al (2018) Diverse mechanisms drive fluoride enrichment in groundwater in two neighboring sites in northern China. Environ Pollut 237:430-441 (in Chinese)

Liu D-S et al (1966) Material composition and structure of loess. Science Presss, BeiJing (in chinese)

Liu D-S, Ch Q-L, Yu Z-C et al (1980) Geochemical environment problems concerning the endemic fluorine disease in China. Geochimica 1:13-22 (in Chinese)

Mao R-Y, Guo H-M, Jia Y-F et al (2016) Distribution characteristics and genesis of fluoride groundwater in the Hetao Basin, Inner Mongolia. Earth Sci Front 23(2):260-268 (in Chinese)

McCaffrey LP, Willis JP (1993) Distribution of fluoride-rich groundwater in the eastern parts of Bophuthatswana, relationship to bedrock and soiis and constraints on drinking water supplies: a preliminary report. Africa Needs Ground Water. An International Ground Water Convention, 8-11

Rafique T (2009) Naseem, S, Usmani T H. Geochemical factors controlling the occurrence of high fluoride groundwater in the Nagar Parkar area, Sindh, Pakistan. J Hazard Mater 171(1/3):424-430
Robertson FN (1984) Solubility controls of fluorine, barium and chromium in ground water in alluvial basins of Arizona. In: B Hitchon and E. I. Wallick, (eds) Proceedings first Canadian/American conference on hydrogeology, Practical applications of ground water geochemistry. National Water Well Association, 96-102

Rukah YA, Alsokhny K (2004) Geochemical assessment of groundwater contamination with special emphasis on fluoride concentration, North Jordan. Chem Erde-Geochem 64(2):171-181

Wu Y, Guo H-M, Han SB et al (1999) Characteristics of fluoride release from aquifer sediments in Xiji of southern Ningxia. Appl Geochem 14:53-65 Hydrogeology \& Engineering Geology 40(5):117-123 (in Chinese)

Wu C, Wu X, Qian C et al (2018) Hydrogeochemistry and groundwater quality assessment of high fluoride levels in the Yanchi endorheic region, northwest China. Appl Geochem 98:404-417 (in Chinese)

Publisher's note Springer Nature remains neutral with regard to jurisdictional claims in published maps and institutional affiliations. 